\title{
Modification of Chitosan Based on Computer Simulation and Its Application as Biomaterial
}

\section{Gurmohinder Heggnnavar}

Submitted date: 28/06/2019 Posted date: 28/06/2019

Licence: CC BY-NC-ND 4.0

Citation information: Heggnnavar, Gurmohinder (2019): Modification of Chitosan Based on Computer Simulation and Its Application as Biomaterial. ChemRxiv. Preprint.

Modification of chitosan has become an important means to obtain chitosan polymers with better properties. In recent years, the application properties of chitosan have been greatly improved by modification, crosslinking, grafting and other modification methods. In order to improve its poor solubility and weak stability and expand its application scope, physical and chemical methods are often used to modify chitosan. As an important natural biomaterial, the modification and application of chitosan have attracted wide attention. This paper briefly summarizes the degradation and modification methods of chitosan at home and abroad and its research results in biomedicine. The interaction of the interface of the knife polymer composite system and the bonding effect of the coupling agent on the interface of the composite were analyzed by computer simulation. The simulation results show that the interaction between the surface and the three polymer segments is the strongest. These results indicate that chitosan is a kind of biomaterial with excellent performance and has broad application prospects.

File list (1)

4218--3889-2--Heggnnavar et al computational chemistr... (152.00 KiB) view on ChemRxiv • download file 


\title{
Modification of Chitosan Based on Computer Simulation and Its Application as Biomaterial
}

\author{
Gurmohinder Heggnnavar
}

Amity Institute of Molecular Medicine \& Stem Cell Research (AIMMSCR), Amity University, Sector125, Noida, 201313, India

\begin{abstract}
Keywords: Computer simulation; Chitosan; Chemical modification; Biomaterials
Abstract. Modification of chitosan has become an important means to obtain chitosan polymers with better properties. In recent years, the application properties of chitosan have been greatly improved by modification, crosslinking, grafting and other modification methods. In order to improve its poor solubility and weak stability and expand its application scope, physical and chemical methods are often used to modify chitosan. As an important natural biomaterial, the modification and application of chitosan have attracted wide attention. This paper briefly summarizes the degradation and modification methods of chitosan at home and abroad and its research results in biomedicine. The interaction of the interface of the knife polymer composite system and the bonding effect of the coupling agent on the interface of the composite were analyzed by computer simulation. The simulation results show that the interaction between the surface and the three polymer segments is the strongest. These results indicate that chitosan is a kind of biomaterial with excellent performance and has broad application prospects.
\end{abstract}

\section{Introduction}

Chitosan is obtained by deacetylation of chitin, and its biosynthesis is second only to cellulose. Chitosan is the only alkaline polysaccharide in natural polysaccharides. It is called the "sixth major factor" in addition to the five major elements of human life essential substances except protein, fat, sugar, vitamins and minerals [1]. Chitin is a carbohydrate, a natural N-acetylated aminopolysaccharide, widely found in nature in low-level biological fungi, algae cells, shells of shrimp, crabs, insects, shells and cartilage of mollusks. , fine plants and so on. It is soluble in organic acids such as acetic acid, and has non-toxicity, biodegradability, good biocompatibility, etc. In addition, there are many amine groups and carboxyl groups in chitosan molecules, which are easy to be chemically modified and modified [2]. In addition, the structure of chitosan is simple and its function is single, which can not meet the higher application requirements. Polymer segments with different functions and compositions can be introduced into chitosan branched chains by grafting modification [3]. Through computer simulation, we can study the effects of the surface microstructures of biomaterials and the external chemical environment on the interaction between biomaterials and organisms at the atomic and molecular level [4]. It provides a valuable theoretical basis for the surface modification of biomaterials with good biocompatibility and bioactivity. Chemical modification of chitosan can improve its physical and chemical properties, broaden the application fields of chitosan and its derivatives, and is one of the hot topics in recent years. China has abundant chitosan resources and superior conditions for production and processing. Therefore, it is of great significance to develop and utilize this resource.

\section{Chemical Modification of Chitosan}

\section{Acylation Reaction}

Chitosan is a product of chitin N-deacetylation, which is second only to cellulose in natural polymers. Its structural formula is shown in Figure 1 below. Chitosan introduces aliphatic or aromatic acyl groups of different molecular weights on macromolecular chains by reaction with acid chlorides or anhydrides [5]. The acylation reaction can be carried out on a hydroxyl group (Oacylation) or an amino group (N-acylation). Chitosan is widely used in the water treatment, biomedical, cosmetic, agricultural and food industries due to its numerous advantages: non-toxic, 
biodegradable, biologically active, biocompatible and antibacterial. Therefore, it is a very interesting research topic to select appropriate conditions and methods to control the degradation of chitosan and prepare chitosan oligosaccharides to expand the application range. Acyl groups in acylated chitosan and its derivatives destroy the hydrogen bond between chitosan and its derivatives, change their crystal structure and improve the solubility of chitosan materials [6]. Computer simulation, as a means of scientific research in addition to experimental research and theoretical research, can study the physical and chemical properties of materials at different time and space scales, from electrons to intermolecular interactions, from micron-sized defects to millimeter-sized bulk materials [7]. Water-soluble $\mathrm{N}$-acetylated chitosan was prepared in ionic liquid aqueous solution. The hygroscopicity and moisture retention properties of the product were studied. The results showed that the product had good hygroscopicity and heat preservation properties.

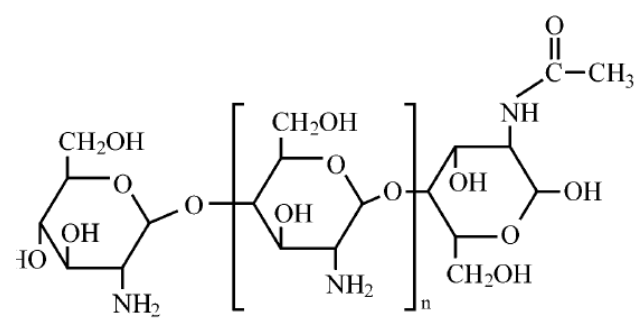

\section{Alkylation Reaction}

Fig. 1 Molecular structure of chitosan

The alkylation reaction generally refers to the reaction of chitosan with a halogenated hydrocarbon or sulphuric acid vinegar to form an alkylated product which can occur either on the carboxyl group or on the amino group. However, since the activity of the amino group is large, the $\mathrm{N}$-alkylation reaction is more likely to occur. This reaction is characterized by the simultaneous introduction of two hydrophilic hydroxyl groups. For example, when chitosan reacts with an excess of an epoxy derivative in an aqueous solution, two $\mathrm{H}$ groups on the molecular amino group are substituted, and the resulting product is easily dissolved. In the water. The results showed that the copolymer had a high adsorption capacity for ampicillin, and the drug release period was as long as $7 \mathrm{~d}$ or more [8]. This is due to the hydrophobic interaction of hydrophobic side chains on the grafts and the interaction of hydrogen bonds between the molecules. It was observed that the hydrogel contained three different states of water: frozen water (free water), non-frozen water (bonded water) and frozen bonded water. Adding nano-materials to chitosan can not only increase the mechanical properties of chitosan, but also change its microstructures. This kind of composite material has a great application in tissue engineering [9]. $\mathrm{N}$-alkylated chitosan was prepared by the reaction of halogenated alkanes with chitosan of different carbon chain lengths. Characterization showed that the intermolecular hydrogen bond and crystallinity of chitosan were weakened after the introduction of alkyl groups, and that the alkylated chitosan cross-linking membrane had no cytotoxicity.

\section{Esterification Modification}

The hydroxyl group of chitosan, especially $\mathrm{C} 6-\mathrm{OH}$, can be esterified with some oxygenated inorganic acids (or their anhydrides). Common vinegarization reactions are sulfated and phosphated. The sulfurization of chitosan is the most attractive area in its chemical modification. In addition, such reactions may also occur on the amino group of chitosan. The acidification reaction of chitosan has been mainly studied by sulfation reaction and phosphation reaction. The use of computer simulation technology to study the surface interface of biomedical materials is superior. It can analyze the role of different parts of this complex system in complex systems, and analyze the macroscopic nature of the speculative system from a microscopic perspective [10]. A polyelectrolyte composite membrane of chitosan and carboxymethylcellulose exhibits good water permeability when used for pervaporation separation of ethanol water. The results show that the modified carboxymethyl chitosan nanoparticles have spherical structure and narrow particle size distribution. Compared with folic acid-chitosan composites, the encapsulation efficiency of modified Carboxymethyl-chitosan particles was significantly improved, and the particle size was significantly reduced. The degradation of chitosan by radioactive radiation can produce physical 
effects such as ionization or excitation, and then produce chemical changes, which can form chemical bond-radiation cross-linking between molecules [11]. Interaction with DNA. The results show that there are two modes of intercalation and electrostatic interaction between sulfated chitosan and fluorescent probe DNA/EB. Sulfated chitosan is a promising target molecule for gene therapy.

On the premise of guaranteeing the simulation accuracy, the simulation samples should be as small as possible. On the other hand, the degree of polymerization of the molecular segments selected by simulation should be large enough. The simulated A-D glycoside bond content of chitosan with $70 \%$ deacetylation degree should be taken as the evaluation index. According to the above method, the number of simulated molecules is determined. The operation results are shown in Figure 2.

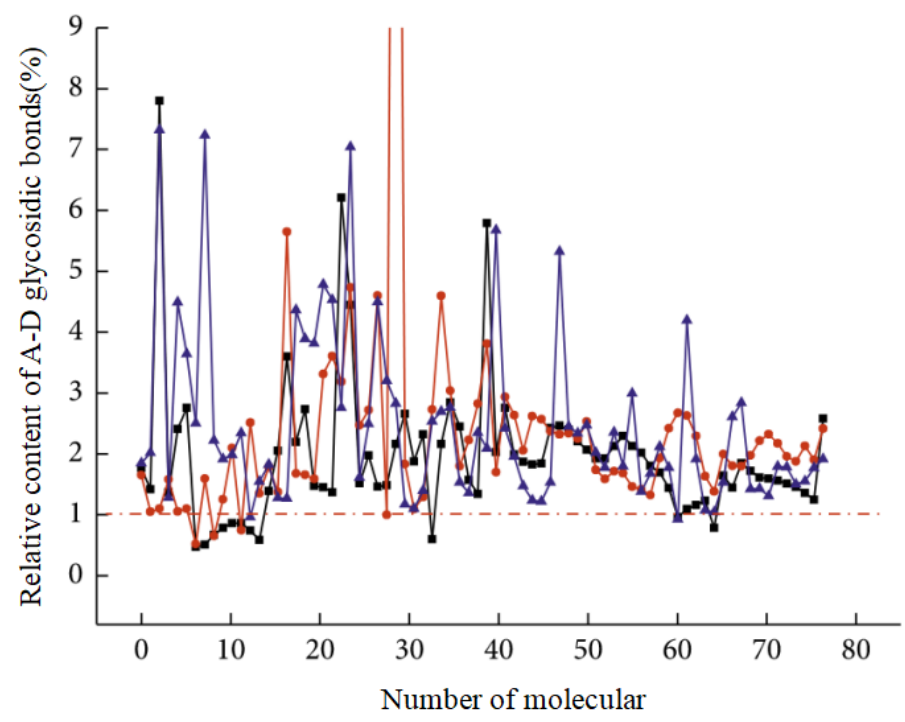

\section{Carboxymethylation Reaction}

Fig. 2 Simulated results of different molecular numbers

The light group or amino group on chitosan reacts with Chloroalkyl acid or glyoxylic acid under different reaction conditions to obtain corresponding carboxylated chitosan derivatives. For example, O-carboxymethyl chitosan can be obtained by the reaction of chitosan with monochloroacetic acid in alkaline condition, and N-carboxymethyl chitosan with glyoxylic acid can be obtained by the reaction of chitosan with glyoxylic acid. A series of hydroxyethyl chitosangrafted polylactic acid copolymers with different compositions were prepared by ring-opening polymerization of racemic lactide initiated by hydroxyethyl chitosan as macromolecular initiator. The grafting rate was greatly increased [12-18]. The basic process of molecular dynamics simulation is to determine the force field parameters for each particle in the research system through a pre-customized force field, determine the initial coordinates and initial velocity of each particle of the system, and calculate the interaction force between the particles. Hydrophobyl modification of carboxymethyl chitosan can be accomplished by two different methods, one by adding a positively charged surfactant and by electrostatic interaction to form a surfactant-polyelectrolyte complex (SPEC) [19 -23]. The second is to introduce a hydrophobic side chain on the amine group of chitosan by an N-alkylation reaction. The results showed that the antibacterial activity of chitosan was obvious; the water solubility of $\mathrm{N}$, O-carboxymethyl chitosan increased and the antibacterial activity decreased.

\section{Graft Copolymerization Modification of Chitosan and Its Application in Biomaterials}

There are many active groups in the molecular chain of chitosan, so the graft copolymerization of chitosan can be carried out on the basis of fully retaining the original skeleton structure of chitosan, so as to improve their properties and meet some special requirements. Because of its good adsorption and selectivity to metal ions and dyes, cross-linked chitosan can be used as a 
concentration and separation agent and wastewater treatment agent. Crosslinking occurs mainly between molecules and is not excluded from the molecule. Chitosan oligosaccharides have been found to exhibit unique functional properties in many ways: they are growth factors of beneficial bacterial bifidobacteria in the intestine; they reduce cholesterol and lipid; and they inhibit the metastasis of some cancer cells. A series of chitosan-polyglutamic acid composite hydrogels were prepared with hydroxyapatite particles as fillers. This hydrogel not only has a high gel content, a low water absorption rate, but also a slow degradation rate. A series of oppositely charged polyelectrolytes can also be formed into ultrafiltration membranes by self-assembly, which can be used for the separation of gases, liquids and ionic compounds. The molecular dynamics simulation makes the research system reach equilibrium, and finally the macroscopic physical properties of interest are calculated according to the model of the equilibrium system. The aggregation phenomenon of chitosan grafted polyethylene glycol copolymer in aqueous solution was studied and analyzed, which indicated that the aggregate structure of the grafted product in aqueous solution could adsorb hydrophobic small molecules in a certain $\mathrm{pH}$ range[34 - 41]. The prepared chitosan grafted styrene-acrylic copolymer emulsion is applied to the sizing of cultural paper, which can improve the printing suitability of the cultural paper.

Due to different degradation methods, different glycosidic bonds on the chitosan molecular chain have different selectivity or degradation ability, and chitosan with different degrees of deacetylation has different glycosidic bond contents in the molecule, which is different. Undoubtedly affect the rate of degradation. Table 1 below shows the relative contents of various glycosidic bonds of chitosan with different degrees of deacetylation.

Table 1 The relative content of various glycosidic bonds of chitosan with different degrees of deacetylation

\begin{tabular}{|c|c|c|c|}
\hline $\begin{array}{c}\text { Degree of } \\
\text { deactylation(DD) } \\
(\%)\end{array}$ & $\begin{array}{c}\text { Relatiye content of } \\
\text { A-D glycosidic } \\
\text { bonds }\left(\mathrm{F}_{\mathrm{A}-\mathrm{D}}\right)(\%)\end{array}$ & $\begin{array}{c}\text { Relative content of } \\
\text { A-A glycosidic } \\
\text { bonds }\left(\mathrm{F}_{\mathrm{A}-\mathrm{A}}\right)(\%)\end{array}$ & $\begin{array}{c}\text { Relative content of } \\
\text { D-D glycosidic } \\
\left.\text { bonds( } \mathrm{F}_{\mathrm{D}-\mathrm{D}}\right)(\%)\end{array}$ \\
\hline 66 & 46 & 24 & 31 \\
\hline 58 & 40 & 33 & 28 \\
\hline
\end{tabular}

The derivative of polyethylene glycol is grafted onto chitosan to form a graft copolymer of both. The entire reaction is divided into two parts: the polyethylene glycol monomethyl ether sodium salt and methyl-chloroacetate are reacted to obtain a carboxymethyl polyethylene glycol. It was found that the composite adsorbent can reach the kinetic adsorption equilibrium at a faster rate for $\mathrm{Ni2+}$. The viscose fiber was used as the substrate and the chitosan was used as the antibacterial agent. The chitosan/viscose fiber blended regenerated fiber was prepared, and the Staphylococcus aureus was used as the test strain [42 - 50]. The results showed that the fiber had deodorant The role of sterilization. Chitosan graft graft copolymers are often used as tissue engineering materials. For example, when chitosan grafted polylactic acid is used as tissue engineering material, it can effectively improve the interaction between cells and the mechanical properties of chitosan. Then it was copolymerized with polycaprolactone ester, and finally the phthalic acid was removed to prepare chitosan-grafted polycaprolactone copolymer. This method is a better tool reaction method for chitosan and hydrophobic polymers. On this basis, the degree of substitution of hydroxypropyl trimethylammonium chloride was determined and the solution properties were studied. The results showed that hydroxypropyl trimethylammonium chloride synthesized by introducing quaternary ammonium salt side chain into the molecular structure of chitosan had good water solubility and high stability.

Chitosan oligosaccharides have excellent water solubility, and their hygroscopicity and moisture retention abilities are significantly higher than those of hyaluronic acid, sodium lactate and glycerol, which can make cosmetics have lubrication and sustained moisture retention. Sodium carboxymethyl chitosan (CMCTS) was synthesized by the reaction of chitosan with chloroacetic acid in the presence of alkali catalyst. Then sodium acrylate and sodium methacrylate were grafted onto CMCTS to prepare water-soluble copolymers. In addition, chitosan can induce immune system to produce antibodies, macrophages, natural killer cells and interferons, and then activate 
macrophages, which directly participate in the activation and regulation of cells, and show anticancer effect. In vitro gene transfection experiments revealed that chitosan-grafted polylysine/deoxyribonucleic acid (pDNA) complexes have higher gene transfection ability to 293T cells than p-DNA and other polymers, chitosan, and PLL. Complex. It not only has good compatibility with cartilage tissue, but also maintains the shape of the tissue. It can be used as a biological scaffold for injectable cartilage regeneration. Among them, when the oppositely charged two component solutions are blended, a unique spherical skin-like macroscopic aggregate is formed, which can be used for coating bioactive substances such as drugs and enzymes. By studying the structural changes of chitosan and its derivatives, the resistance of chitosan derivatives to staphylococci and Escherichia coli was also explored by live cell cytometry. The test showed that this derivative has good antibacterial ability.

\section{Summary}

Chitosan and its derivatives are attracting more and more attention as researchers, and in recent years, a large number of studies on chitosan modification and application as biomaterials have been made at home and abroad. Chitosan can be graft modified by various polymerization methods such as active controlled polymerization, and various graft copolymers with various structures and compositions can be obtained, which opens up a new direction for the functionalization of chitosan. It also opens up new avenues for the application of chitosan, a natural biomaterial. Because of its excellent performance, abundant storage, and special molecular structure, it can be easily modified to make its mechanical and biological properties more widely and better regulated. The active amino and hydroxyl groups on chitosan can be used to introduce new groups through modification, crosslinking, grafting and other modification methods. The graft copolymers with improved properties can be obtained, which has important scientific research value and economic value. Such as medicine, environmental protection, food, daily chemical, agriculture and so on. At the same time, chitosan has a wide range of sources, cheap and easy to obtain, and its development and application prospects will be very broad.

\section{References}

[1] A. Aartsma-Rus, FDA approval of nusinersen for spinal muscular atrophy makes 2016 the year of splice modulating oligonucleotides, Nucleic acid therapeutics, 27 (2017) 67-69.

[2] A. Akinc, A. Zumbuehl, M. Goldberg, E.S. Leshchiner, V. Busini, N. Hossain, S.A. Bacallado, D.N. Nguyen, J. Fuller, R. Alvarez, A combinatorial library of lipid-like materials for delivery of RNAi therapeutics, Nature biotechnology, 26 (2008) 561.

[3] M. An, C. Yu, J. Xi, J. Reyes, G. Mao, W.-Z. Wei, H. Liu, Induction of necrotic cell death and activation of STING in the tumor microenvironment via cationic silica nanoparticles leading to enhanced antitumor immunity, Nanoscale, 10 (2018) 9311-9319.

[4] R.L. Ball, P. Bajaj, K.A. Whitehead, Oral delivery of siRNA lipid nanoparticles: Fate in the GI tract, Scientific reports, 8 (2018) 2178.

[5] E. Blanco, H. Shen, M. Ferrari, Principles of nanoparticle design for overcoming biological barriers to drug delivery, Nature biotechnology, 33 (2015) 941.

[6] H.-I. Chang, M.-K. Yeh, Clinical development of liposome-based drugs: formulation, characterization, and therapeutic efficacy, International journal of nanomedicine, 7 (2012) 49. 
[7] Y. Cheng, H. Wei, J.K.Y. Tan, D.J. Peeler, D.O. Maris, D.L. Sellers, P.J. Horner, S.H. Pun, NanoSized Sunflower Polycations As Effective Gene Transfer Vehicles, Small, 12 (2016) 2750-2758.

[8] D. Christensen, K.S. Korsholm, P. Andersen, E.M. Agger, Cationic liposomes as vaccine adjuvants, Expert review of vaccines, 10 (2011) 513-521.

[9] R. Zhang, J.D. Smith, B.N. Allen, J.S. Kramer, M. Schauflinger, B.D. Ulery, Peptide Amphiphile Micelle Vaccine Size and Charge Influence the Host Antibody Response, ACS Biomaterials Science \& Engineering, 4 (2018) 2463-2472.

[10] R. Zhang, B.D. Ulery, Synthetic vaccine characterization and design, Journal of Bionanoscience, 12 (2018) 1-11.

[11] A. Wroblewska, M. Dhainaut, B. Ben-Zvi, S.A. Rose, E.S. Park, E.-A.D. Amir, A. Bektesevic, A. Baccarini, M. Merad, A.H. Rahman, Protein Barcodes Enable High-Dimensional Single-Cell CRISPR Screens, Cell, 175 (2018) 1141-1155. e1116.

[12] R. Xavier, D. Podolsky, Unravelling the pathogenesis of inflammatory bowel disease, Nature, 448 (2007) 427.

[13] Z. Yaari, D. Da Silva, A. Zinger, E. Goldman, A. Kajal, R. Tshuva, E. Barak, N. Dahan, D. Hershkovitz, M. Goldfeder, Theranostic barcoded nanoparticles for personalized cancer medicine, Nature communications, 7 (2016) 13325.

[14] R. Zhang, L.D. Morton, J.D. Smith, F. Gallazzi, T.A. White, B.D. Ulery, Instructive Design of Triblock Peptide Amphiphiles for Structurally Complex Micelle Fabrication, ACS Biomaterials Science \& Engineering, (2018).

[15] J.D. Smith, L.N. Cardwell, D. Porciani, J.A. Nguyen, R. Zhang, F. Gallazzi, R.R. Tata, D.H. Burke, M.A. Daniels, B.D. Ulery, Aptamer-displaying peptide amphiphile micelles as a celltargeted delivery vehicle of peptide cargoes, Physical biology, 15 (2018) 065006.

[16] K.M. Takeda, K. Osada, T.A. Tockary, A. Dirisala, Q. Chen, K. Kataoka, Poly (ethylene glycol) crowding as critical factor to determine pDNA packaging scheme into polyplex micelles for enhanced gene expression, Biomacromolecules, 18 (2016) 36-43.

[17] B. Weide, S. Pascolo, B. Scheel, E. Derhovanessian, A. Pflugfelder, T.K. Eigentler, G. Pawelec, I. Hoerr, H.-G. Rammensee, C. Garbe, Direct injection of protamine-protected mRNA: results of a phase $1 / 2$ vaccination trial in metastatic melanoma patients, Journal of immunotherapy, 32 (2009) 498-507.

[18] K.A. Whitehead, J.R. Dorkin, A.J. Vegas, P.H. Chang, O. Veiseh, J. Matthews, O.S. Fenton, Y. Zhang, K.T. Olejnik, V. Yesilyurt, Degradable lipid nanoparticles with predictable in vivo siRNA delivery activity, Nature communications, 5 (2014) 4277.

[19] N. Pardi, M.J. Hogan, F.W. Porter, D. Weissman, mRNA vaccines—a new era in vaccinology, Nature Reviews Drug Discovery, 17 (2018) 261. 
[20] S. Patel, N. Ashwanikumar, E. Robinson, A. DuRoss, C. Sun, K.E. Murphy-Benenato, C. Mihai, O.r. Almarsson, G. Sahay, Boosting intracellular delivery of lipid nanoparticle-encapsulated mRNA, Nano letters, 17 (2017) 5711-5718.

[21] K. Paunovska, A.J. Da Silva Sanchez, C.D. Sago, Z. Gan, M.P. Lokugamage, F.Z. Islam, S. Kalathoor, B.R. Krupczak, J.E. Dahlman, Nanoparticles Containing Oxidized Cholesterol Deliver mRNA to the Liver Microenvironment at Clinically Relevant Doses, Advanced Materials, (2019) 1807748.

[22] E. Robinson, K.D. MacDonald, K. Slaughter, M. McKinney, S. Patel, C. Sun, G. Sahay, Lipid Nanoparticle-Delivered Chemically Modified mRNA Restores Chloride Secretion in Cystic Fibrosis, Molecular Therapy, (2018).

[23] C.D. Sago, M. Lokugamage, G.N. Lando, N. Djeddar, N. Shah, C. Syed, A.V. Bryksin, J.E. Dahlman, Modifying a commonly expressed endocytic receptor retargets nanoparticles in vivo, Nano letters, (2018).

[24] C.D. Sago, M.P. Lokugamage, K. Paunovska, D.A. Vanover, C.M. Monaco, N.N. Shah, M.G. Castro, S.E. Anderson, T.G. Rudoltz, G.N. Lando, High-throughput in vivo screen of functional mRNA delivery identifies nanoparticles for endothelial cell gene editing, Proceedings of the National Academy of Sciences, 115 (2018) E9944-E9952.

[25] A.J. Mukalel, R.S. Riley, R. Zhang, M.J. Mitchell, Nanoparticles for nucleic acid delivery: Applications in cancer immunotherapy, Cancer letters, 458 (2019) 102-112.

[26] R. Zhang, M.M. Billingsley, M.J. Mitchell, Biomaterials for vaccine-based cancer immunotherapy, Journal of Controlled Release, (2018).

[27] R. Zhang, J.S. Kramer, J.D. Smith, B.N. Allen, C.N. Leeper, X. Li, L.D. Morton, F. Gallazzi, B.D. Ulery, Vaccine Adjuvant Incorporation Strategy Dictates Peptide Amphiphile Micelle Immunostimulatory Capacity, The AAPS journal, 20 (2018) 73.

[28] J. Karlsson, H.J. Vaughan, J.J. Green, Biodegradable Polymeric Nanoparticles for Therapeutic Cancer Treatments, Annual review of chemical and biomolecular engineering, (2018).

[29] K.J. Kauffman, J.R. Dorkin, J.H. Yang, M.W. Heartlein, F. DeRosa, F.F. Mir, O.S. Fenton, D.G. Anderson, Optimization of lipid nanoparticle formulations for mRNA delivery in vivo with fractional factorial and definitive screening designs, Nano letters, 15 (2015) 7300-7306.

[30] R. Zhang, C.N. Leeper, X. Wang, T.A. White, B.D. Ulery, Immunomodulatory vasoactive intestinal peptide amphiphile micelles, Biomaterials science, 6 (2018) 1717-1722.

[31] R. Kole, A.R. Krainer, S. Altman, RNA therapeutics: beyond RNA interference and antisense oligonucleotides, Nature reviews Drug discovery, 11 (2012) 125.

[32] L.M. Kranz, M. Diken, H. Haas, S. Kreiter, C. Loquai, K.C. Reuter, M. Meng, D. Fritz, F. Vascotto, H. Hefesha, Systemic RNA delivery to dendritic cells exploits antiviral defence for cancer immunotherapy, Nature, 534 (2016) 396. 
[33] A.M. Krieg, A.-K. Yi, S. Matson, T.J. Waldschmidt, G.A. Bishop, R. Teasdale, G.A. Koretzky, D.M. Klinman, CpG motifs in bacterial DNA trigger direct B-cell activation, Nature, 374 (1995) 546.

[34] C. Kriegel, H. Attarwala, M. Amiji, Multi-compartmental oral delivery systems for nucleic acid therapy in the gastrointestinal tract, Advanced drug delivery reviews, 65 (2013) 891-901.

[35] J.A. Kulkarni, J.L. Myhre, S. Chen, Y.Y.C. Tam, A. Danescu, J.M. Richman, P.R. Cullis, Design of lipid nanoparticles for in vitro and in vivo delivery of plasmid DNA, Nanomedicine: Nanotechnology, Biology and Medicine, 13 (2017) 1377-1387.

[36] A.K. Leung, I.M. Hafez, S. Baoukina, N.M. Belliveau, I.V. Zhigaltsev, E. Afshinmanesh, D.P. Tieleman, C.L. Hansen, M.J. Hope, P.R. Cullis, Lipid nanoparticles containing siRNA synthesized by microfluidic mixing exhibit an electron-dense nanostructured core, The Journal of Physical Chemistry C, 116 (2012) 18440-18450.

[37] B. Li, Y. Dong, Preparation and optimization of lipid-like nanoparticles for mRNA delivery, in: RNA Nanostructures, Springer, 2017, pp. 207-217.

[38] X. Luo, B. Li, X. Zhang, W. Zhao, A. Bratasz, B. Deng, D. McComb, Y. Dong, Dual-functional lipid-like nanoparticles for delivery of mRNA and MRI contrast agents, Nanoscale, 9 (2017) 15751579.

[39] P. Midoux, C. Pichon, Lipid-based mRNA vaccine delivery systems, Expert review of vaccines, 14 (2015) 221-234.

[40] M.J. Mitchell, R.K. Jain, R. Langer, Engineering and physical sciences in oncology: challenges and opportunities, Nature Reviews Cancer, 17 (2017) 659.

[41] K. Halder, M. Benzler, J.S. Hartig, Reporter assays for studying quadruplex nucleic acids, Methods, 57 (2012) 115-121.

[42] D. Ibraheem, A. Elaissari, H. Fessi, Gene therapy and DNA delivery systems, International journal of pharmaceutics, 459 (2014) 70-83.

[43] R. Kanasty, J.R. Dorkin, A. Vegas, D. Anderson, Delivery materials for siRNA therapeutics, Nature materials, 12 (2013) 967.

[44] P.W. Laird, A. Zijderveld, K. Linders, M.A. Rudnicki, R. Jaenisch, A. Berns, Simplified mammalian DNA isolation procedure, Nucleic acids research, 19 (1991) 4293. 
4218--3889-2--Heggnnavar et al computational chemistr... (152.00 KiB) view on ChemRxiv • download file 